\title{
Knowing patients' preferences for place of death: how possible or desirable?
}

In their paper in this issue of the BJGP, Meeussen et al report that GPs knew the preferred place of death for $46 \%$ of their patients with non-sudden deaths. This information came from the patient alone for $40 \%$, from significant others alone for $36 \%$ and from both for $22 \%$. Patients whose GP knew their preferences were more likely to achieve their wish, and GP knowledge was associated with greater GP involvement in end-of-life care. The authors call for improvement in GPs' knowledge of patients' preferences for place of death, suggesting this to be a marker of end-of-life care quality.

The majority of terminally ill patients prefer to die at home; a preference that declines as illness progresses., ${ }^{2,3}$ However, such a preference is neither categorical nor fixed, but 'a socially contingent leaning in a particular direction, rather than an abstractly arrived at certainty' qualified by speculation about how things might change with events. ${ }^{4} \mathrm{~A}$ recent paper in the $B M J^{5}$ found preferences for place of death to be often poorly formed in patients' minds, and often inferred by health professionals without direct questioning or reaching a definitive answer. The enabling of patients to achieve their preferred place of death is viewed by the authors as a very limited proxy measure for the effectiveness of palliative care delivery.

The NHS End of Life Care Strategy ${ }^{6}$ advocates a care pathway that starts with health professionals discussing with patients their care preferences, and advocates the use of the Preferred Priorities for Care document. ${ }^{7}$ This is a welcome challenge to health professionals: all of us at times fail to provide adequate opportunities for discussion, or block such conversations when they arise. However, exploring preferences with patients is challenging at several levels.
- The GP needs to recognise the probable approach of life's end. This is increasingly difficult in cancer where the dying trajectory ${ }^{8}$ has been moderated to one more akin to a chronic disease, due to its therapeutic advances and the increase in continuation of such treatment close to death. Accurate prognostication is very difficult, if not impossible, in the fluctuating dying trajectory of non-malignant illness. It would greatly help if our specialist colleagues either started these discussions with patients themselves, or set the scene for the GP to do so. A GP often lacks the specialist knowledge needed concerning new treatments to inform the conversation.

- The patient needs to accept the possible approach of life's end. Noncancer patients frequently see their illness as chronic rather than lifelimiting, ${ }^{9}$ and may be shocked to be told otherwise. While it is true that denial is an ego defence mechanism that must be respected, it also prevents the open communication that many view as key to 'a good death'.

- The GP needs to be comfortable with discussing end-of-life issues. The current reluctance to hold such discussions reminds one of the medical profession's reluctance 20 years ago to tell patients of a cancer diagnosis. An established and trusting relationship with the patients and family is essential, as is confidence that services will reliably be available when needed. It is so easy to move the conversation away from what may lie ahead onto the safer territory of drugs and doses. Should a GP feel unable to discuss these matters with their patients, they may need to involve a colleague who is more comfortable in the area, such as a fellow GP, community nurse, or palliative care specialist.

- The patient needs to be comfortable talking about their preferences and views. They may not wish to talk now, not with that GP, not with any professional, or never with anyone. Or they may very much want to raise these issues, but just not know how to start the conversation. Patients often wait for doctors to open up the conversation, while their doctors wait for patients to raise the issue.

Judging the appropriate time to raise end-of-life issues with patients and their families is very challenging. Are we reaching that stage in the disease process? Too soon might demotivate patients, causing them to 'give up'. Too late (currently a common situation) does not allow patient-centred plans to be made. Time and high-level communication skills honed by experience are needed gently to indicate to the patient a willingness to discuss what their future wishes might be should things get worse; and then to allow the patient either to discuss issues should they wish, or to respect their reluctance to discuss the issue. We cannot impose timing or content: not only are patients' preferences for place of death very individual, so are their preferences for whether and when to talk, and with whom.

It is not a failure if a patient dies having never discussed their preference for place of death, provided that opportunities have been repeatedly been provided to do so: it is their final illness. Care needs to be handled in the way that they prefer, even if it is untidy and creates uncertainty for the GP and others involved in their care. A preferred priority for silence is to be respected.

\section{Stephen Barclay,}

Macmillan Post-Doctoral Research Fellow, General Practice and Primary Care Research Unit, University of Cambridge. Member of the NIHR CLAHRC for Cambridgeshire and Peterborough (Collaboration for Leadership in Applied Health Research and Care) 


\section{Steve Case-Upton}

Academic GP Registrar, General Practice and Primary Care Research Unit, University of Cambridge.

\section{Provenance}

Commissioned; not peer reviewed.

\section{REFERENCES}

1. Meeussen K, Van den Block L, Bossuyt N. GPs' awareness of patients' preference for place of death. $\mathrm{Br} \mathrm{J}$ Gen Pract 2009; 59(566): 665-670.

2. Townsend J, Frank AO, Fermont D, et al. Terminal cancer care and patients' preference for place of death: a prospective study. BMJ 1990; 301(6749): 415-417.

3. Higginson IJ, Sen-Gupta GJ. Place of care in advanced cancer: a qualitative systematic literature review of patient preferences. J Palliat Med 2000; 3(3): 287-300.

4. Thomas C, Morris SM, Clark D. Place of death: preferences among cancer patients and their carers. Soc Sci Med 2004; 58(12): 2431-2444.

5. Munday D, Petrova M, Dale J. Exploring preferences for place of death with terminally ill patients: qualitative study of experiences of general practitioners and community nurses in England. BMJ 2009; 339: 214-218

6. Department of Health. End of Life Care Strategy: promoting high quality care for all adults at the end of life. London; Department of Health, 2008.

7. Storey L. The Preferred Priorities for Care (PPC) document: guidelines for health and/or social care staff. Leicester: National End of Life Care Programme, 2007. http://www endoflifecareforadults nhs uk/eolc/ppc htm (accessed 5 Aug 2009).

8. Lunney JR, Lynn J, Foley DJ, et al. Patterns of functional decline at the end of life. JAMA 2003; 289(18): 2387-2392.

9. Murray SA, Boyd K, Kendall M, et al. Dying of lung cancer or cardiac failure: prospective qualitative interview study of patients and their carers in the community. BMJ 2002; 325(7370): 929-932.

DOI: 10.3399/bjgp09X454052

\section{ADDRESS FOR CORRESPONDENCE}

\section{Stephen Barclay}

University of Cambridge, General Practice and Primary Care Research Unit, Department of Public Health and Primary Care, Institute of Public Health, Robinson Way, Cambridge, CB2 0SR, UK E-mail: sigb2@medschl.cam.ac.uk 